\title{
The Embodiment of Emotional Feelings in the Brain
}

\author{
Neil A. Harrison, ${ }^{1,3}$ Marcus A. Gray, ${ }^{1}$ Peter J. Gianaros, ${ }^{4}$ and Hugo D. Critchley ${ }^{1,2,3}$ \\ ${ }^{1}$ Department of Psychiatry, Brighton and Sussex Medical School, and ${ }^{2}$ Sackler Centre for Consciousness Science, University of Sussex, Brighton BN1 9PR, \\ United Kingdom, ${ }^{3}$ Sussex Partnership Foundation Trust, Worthing, West Sussex BN13 3EP, United Kingdom, and ${ }^{4}$ Department of Psychiatry, University of \\ Pittsburgh, Pittsburgh, Pennsylvania, 15213
}

Central to Walter Cannon's challenge to peripheral theories of emotion was that bodily arousal responses are too undifferentiated to account for the wealth of emotional feelings. Despite considerable evidence to the contrary, this remains widely accepted and for nearly a century has left the issue of whether visceral afferent signals are essential for emotional experience unresolved. Here we combine functional magnetic resonance imaging and multiorgan physiological recording to dissect experience of two distinct disgust forms and their relationship to peripheral and central physiological activity. We show that experience of core and bodyboundary-violation disgust are dissociable in both peripheral autonomic and central neural responses and also that emotional experience specific to anterior insular activity encodes these different underlying patterns of peripheral physiological responses. These findings demonstrate that organ-specific physiological responses differentiate emotional feeling states and support the hypothesis that central representations of organism physiological homeostasis constitute a critical aspect of the neural basis of feelings.

\section{Introduction}

Do we run from a bear because we are afraid or are we afraid because we run? William James posed this question more than a century ago (James, 1894), yet the notion that afferent visceral signals are essential for the unique experiences of distinct emotions remains a key unresolved question at the heart of emotional neuroscience (Cacioppo et al., 2000; Rainville et al., 2006). Early challenges to the James-Lange position, that emotional feelings result from central representations of changes in body physiology, rested on beliefs that autonomic outflow was insufficiently differentiated to enable emotion-specific response patterns (Cannon, 1931). Demonstration that both sympathetic (Morrison, 2001; Janig, 2008) and parasympathetic (Porges, 2007) nervous systems possess exquisite organ-specific regulation has countered this argument and contributed to a revival of somatic theories (Damasio, 1994).

For example, emotion-specific patterns of peripheral autonomic activity have been reported to static visual (Collet et al., 1997), olfactory (Vernet-Maury et al., 1999), and dynamic (Christie and Friedman, 2004; Rainville et al., 2006) emotional stimuli to voluntarily produced emotional facial expressions (Ekman et al., 1983; Levenson et al., 1990) and experiential reliving of emotion (Ekman et al., 1983; Rainville et al., 2006). Similarly, emotion-specific patterns of neural activity have been demonstrated in association with discrete feeling states (Damasio et al.,

\footnotetext{
Received April 5, 2010; revised Aug. 4, 2010; accepted Aug. 11, 2010.

This study was supported by grants from the Wellcome Trust Programme to H.D.C. and from the Wellcome Trust Centre to the Wellcome Trust Centre for Neuroimaging.

The authors declare no competing financial interests.

Correspondence should be addressed to Neil A. Harrison, Clinical Imaging Sciences Centre, Brighton and Sussex

Medical School, University of Sussex, Brighton BN1 9RR, UK. E-mail: n.harrison@bsms.ac.uk.

DOI:10.1523/JNEUROSCI.1725-10.2010

Copyright $\odot 2010$ the authors $\quad 0270-6474 / 10 / 3012878-07 \$ 15.00 / 0$
}

2000). Critically, however, methodological difficulties in integrating central neural and peripheral physiological response recording with subjective experience has left the issue of whether unique physiological patterning contributes to emotion-specific patterns of neural activity and associated feeling states unresolved (Cacioppo et al., 2000). Here we address this issue through a unique combination of functional magnetic resonance imaging (fMRI) and simultaneous recording of autonomic influences on two independent organ systems (heart and stomach) during the experience of two different forms of disgust. Gastric myoelectric responses, in particular, were selected because of their described sensitivity to feelings of nausea (Stern, 2002) and disgust (Jokerst et al., 1997).

Anatomical studies have demonstrated a class of interoceptive afferent fibers that monitor the physiological state of all internal organs (Andrew and Craig, 2001a). These lamina I afferents project via brainstem nuclei (Andrew and Craig, 2001b; Craig, 2002) and dedicated thalamic nuclei to dorsal posterior insula bilaterally. Here, it is proposed, they provide a highly resolved, somatotopically organized cortical representation of the physiological condition of all bodily tissues (Damasio et al., 2000; Craig, 2002) and support a constellation of motivationally significant bodily sensations including temperature, pain, itch, muscular and visceral ache, and sensual touch (Craig, 2002). Iterative (posterior to anterior) remapping of these representations along insula cortex is proposed to enable progressive incorporation with multimodal information concerning emotionally salient environmental stimuli. Anterior insula, which has connections with amygdala, cingulate, ventral striatum, and prefrontal and multimodal sensory regions (Mufson and Mesulam, 1982), is believed ultimately to the integrate homeostatic state with motivational, hedonic, cognitive, and social signals represented in other brain regions (Craig, 2002) and support associated feeling states. Func- 
tional neuroimaging studies support this experiential role of anterior insula showing activation to interoceptive awareness (Critchley et al., 2004) and feelings as diverse as anger (Damasio et al., 2000), sense of knowing (Kikyo et al., 2002), disgust (Phillips et al., 1997), and trustworthiness (Craig, 2002; Winston et al., 2002). Similarly, studies of individuals with insula lesions have shown impairments in emotion recognition and experience, particularly disgust (Jones et al., 2010), and an acute reduction in conscious urges to smoke associated with an ability to quit smoking easily and to remain abstinent (Naqvi et al., 2007).

This model, however, also makes another key and, to date, untested prediction: if cortical representations of homeostatic physiological activity form the basis for subjective emotional experiences, and anterior insula awareness of these feelings, then emotion-related anterior insular activity will also encode the underlying patterns of peripheral physiological responses. This may be apparent in firing characteristics of neural ensembles or in differential topographical segregation within anterior insula. By simultaneously recording fMRI and autonomic influences on two independent internal organs, we sought to test this prediction and investigate how emotion-specific physiological patterning may contribute to emotion-dependent patterns of neural activity and underpin differences in associated feeling states.

\section{Materials and Methods}

Subjects. Participants were 12 healthy subjects [seven females; mean age ( \pm SD), 25.9 ( \pm 5.7 ) years]. All were right handed, had normal or corrected-to-normal vision, no structural brain abnormality, and no past neurological or psychiatric history. All denied drug use within the last 6 months. Subjects were instructed to eat a standard breakfast of two slices of toast and jam and one glass of orange juice 45-60 min before arrival in the laboratory. Informed consent was obtained in accordance with the Declaration of Helsinki (1991), and the procedures were approved by the joint University College London/University College London Hospitals Ethics Committee. Participants were recruited by advertisement on a departmental website and given a small financial reimbursement for involvement in the study.

Stimuli and behavioral data analysis. Stimuli were specially created 2-min-duration color videos designed to induce potent feelings of disgust. Body-boundary-violation (BBV) disgust videos were produced using edited clips of surgical operations taken from a surgical training archive. Videos designed to induce core ingestive disgust were created using two actors (one male and one female). They were filmed looking at, smelling, eating, and mock vomiting a mixture of foul smelling and visually repulsive (though edible) food. Four additional low-intensity control videos were also produced to play between the potent disgustinducing videos. Further details of each of the stimuli can be found in the supplemental material (available at www.jneurosci.org).

Subjects viewed each video in random order while undergoing fMRI in two sessions. Half of the videos were shown in the first session, and half were shown in the second session. After each video, subjects used a visual analog scale to indicate how disgusted, light-headed/faint, and nauseated each video made them feel. After rating the stimuli, a blank white screen was displayed to ensure videos were separated by a minimum of $30 \mathrm{~s}$. Rating responses were indicated using an on-screen cursor controlled via a right-hand button-box. Group mean ratings of induced disgust were then used to obtain experienced disgust ratings for each video and, additionally, to separate both video types into high- and low-disgust groups with three videos in each group. This enabled all subsequent peripheral physiological (gastric and cardiac) and fMRI data to be analyzed using repeated-measures ANOVA adopting a factorial approach with the two factors: disgust modality (BBV and core disgust) and experienced disgust intensity.

Scanning procedure and imaging data analysis. Whole-brain $\mathrm{fMRI}$ data were acquired on a 3.0T Siemens Allegra magnetic resonance scanner equipped with a standard head coil. Functional images were obtained with a gradient echo-planar $\mathrm{T}^{\star}$ sequence using blood-oxygenation level-dependent contrast, each comprising a full brain volume of 48 contiguous slices ( $2 \mathrm{~mm}$ slice thickness, $1 \mathrm{~mm}$ interslice gap) in a $-30^{\circ}$ tilted plane acquisition sequence to minimize signal dropout in the orbitofrontal, medial temporal, and brainstem regions (Deichmann et al., 2003). Volumes were acquired continuously with a repetition time of $3.12 \mathrm{~s}$. Three hundred eighty-two volumes were acquired for each participant per session (19.8 min), with the first five volumes of each session subsequently discarded to allow for T1 equilibration effects. Between sessions, subjects were taken out of the scanner and asked to drink $500 \mathrm{ml}$ of water at room temperature to maintain gastric activity. Field maps were acquired at the end of each session to enable subsequent unwarping of functional data with regard to the B0 field. Finally, a high-definition T1-weighted anatomical scan was also acquired.

fMRI data were analyzed as a block design in SPM5 (Wellcome Department of Imaging Neuroscience; www.fil.ion.ucl.ac.uk/spm). Individual scans were realigned and unwarped, time corrected, normalized, and spatially smoothed with an $8 \mathrm{~mm}$ full-width at half-maximum Gaussian kernel. A high-pass frequency filter (cutoff, $128 \mathrm{~s}$ ) was applied to the time series. Each video was modeled separately as a 2 min block convolved with a standard synthetic hemodynamic response function. Parameter estimates of block-related activity were obtained at each voxel, for each individual video and subject. Statistical parametric maps of the $t$ statistic (SPM $\{t\})$ were then generated for the following contrasts: (1) observation of core disgust videos, (2) observation of BBV disgust videos, (3) experience of core disgust, and (4) experience of BBV disgust. Contrasts 3 and 4, which enabled direct comparison with the physiological data, were obtained by parametric modulation of contrasts 1 and 2 by mean disgust ratings to core and BBV videos, respectively.

In addition, after separating the videos into high and low experienced disgust groups, the following contrasts were also obtained: low core disgust, high core disgust, low BBV disgust, and high BBV disgust. Contrasts were then transformed to a normal distribution $(\operatorname{SPM}\{Z\})$ for each individual participant. Second-level random-effects analyses (subjects as random variable) were then performed using repeated-measures ANOVAs on the contrast images obtained for each of the $2 \times 2$ factor combinations for each subject. Results are reported for all insula voxels surviving a threshold of $p<0.001$, and extra-insula activations are only reported for gray matter clusters of $\geq 10$ contiguous voxels. Group-level correlations of peripheral physiological responses and contrast images to each video in both modalities were also subsequently performed.

Physiological data recording. Cardiac data were recorded using pulse oximetry recorded at $500 \mathrm{~Hz}$. Gastric electrogastrogram (EGG) was recorded using four pairs of MRI-compatible ceramic mounted silver/ silver-chloride electrodes placed on the abdominal midline $2-3 \mathrm{~cm}$ above the umbilicus and $\sim 6 \mathrm{~cm}$ to the left and $3-4 \mathrm{~cm}$ superior to the midline electrode (Gianaros et al., 2001). A reference electrode was positioned 10 $\mathrm{cm}$ to the right of the midline and $3 \mathrm{~cm}$ above the umbilicus. Myoelectric signals were amplified using an MRI-compatible, shielded, nonmagnetic, battery-operated constant current amplifier (Brain Products BrainAmp ExG MR; input impedance, $10 \mathrm{G} \Omega$; input noise, $1 \mu \mathrm{Vpp}$; common mode rejection, $120 \mathrm{~dB}$ ). The EGG signal was digitized at $5 \mathrm{kHz}$ per channel. Amplifier output was via twin fiber optic channels to a laptop running Analyzer software.

EGG signals were recorded on a laptop running Analyzer software. Pulse oximeter waveform and scanner pulse signals were passed to a Cambridge Electronic Design Power1401 data acquisition interface and recorded on a separate computer running Spike2 software. Computers running Spike2 and Analyzer were synchronized with the stimulus computer playing the videos and recording subject responses via a common timing pulse from the stimulus computer.

Analysis of physiological data. According to the literature, heart rate data recorded using a pulse oximeter (Nonin 8600; Nonin Medical) on the left index finger were analyzed according to the following frequency bands: high frequency (parasympathetic), 0.150-0.394 Hz; low frequency (sympathetic), $0.039-0.146 \mathrm{~Hz}$; very low frequency (VLF; uncertain, thermal regulation), $0.006-0.033 \mathrm{~Hz}$.

All physiological data were analyzed in Matlab using purpose-written routines. Cardiac contractions were identified from maxima in the pulse 
oximeter waveform data and used to determine successive R-R intervals. $\mathrm{R}-\mathrm{R}$ intervals were then visually inspected to identify infrequent missed beats or ventricular ectopics, which were replaced by interpolation with neighboring $\mathrm{R}-\mathrm{R}$ intervals. The $\mathrm{R}-\mathrm{R}$ intervals were then interpolated in time to produce equal interval interbeat-interval (IBI) time series and downsampled to $64 \mathrm{~Hz}$. Frequency domain analyses were then performed on 3 min segments of IBI data (overlapping the video playback by \pm 30 s). IBI data were Hamming windowed and forward and reverse filtered with third-order Butterworth filters ([0.01 1.0 $] / 32)$ before undergoing fast Fourier transform (FFT). Spectral densities were derived in $0.33 \mathrm{cpm}$ bins within the frequency range of $0.006-0.394 \mathrm{~Hz}$. The percentage of total power (square of the real component of the FFT) within this range for high- and low-frequency components was then calculated using the following equations: \% High frequency $=(0.150-0.394 \mathrm{~Hz}) /$ $(0.006-0.394 \mathrm{~Hz}$ power $), \%$ Low frequency $=(0.039-0.146 \mathrm{~Hz}) /$ $(0.006-0.394 \mathrm{~Hz}$ power). Inclusion of VLF power within the denominator enabled us to determine both low frequency and high frequency relative power. The percentage of total power within the VLF band, however, was not used in subsequent analyses given that physiological interpretation of changes within this frequency band remain poorly understood (Berntson et al., 1997).

Analysis of all of the EGG data was performed in Matlab using purpose-written routines. Spectral analyses (frequency domain) using discrete FFTs were performed on 3 min epochs of EGG data (overlapping the video playback by $\pm 30 \mathrm{~s})$. Before spectral analysis, the EGG time series for each epoch was linearly detrended (to remove slow drifts in signal), mean centered, and downsampled to $5 \mathrm{~Hz}$. A Hamming window was then used to taper the EGG signal. After windowing, the data were forward and reverse filtered with a third-order Butterworth filter ([1/60 $12 / 60] / 2.5]$ ) before undergoing FFT. Spectral densities were derived in $0.33 \mathrm{cpm}$ bins within the frequency range of $0.33-9.66 \mathrm{cpm}$. The percentage of total power (square of the real component of the FFT) was calculated for tachygastria $(4.00-9.66 \mathrm{cpm})$, normogastria $(2.66-3.66 \mathrm{cpm})$, and bradygastria $(0.33-2.33 \mathrm{cpm})$ using the following equations: \% tachygastria $=(4.00-9.66 \mathrm{cpm}$ power $) /(0.33-9.66 \mathrm{cpm}$ power $), \%$ normogastria $=(2.66-3.66 \mathrm{cpm}$ power $) /(0.33-9.66 \mathrm{cpm}$ power $), \%$ bradygastria $(0.33-2.33 \mathrm{cpm}$ power $) /(0.33-9.66 \mathrm{cpm}$ power $)$.

Subject-specific percentage power for gastric (bradygastric, normogastric, and tachygastric power) and cardiac (high, low, and VLF power) responses were then determined to each video and used to produce group mean physiological responses in each band for each video. Mean cardiac (high and low frequency) and gastric (normogastric and tachygastric) physiological responses to each video were then used as covariates in a general linear model with disgust type (BBV or core) as a random factor to determine physiological predictors of differential feelings of disgust. Interactions of disgust type and high and low cardiac power and tachygastria were included in the model. Visualization of the relationship between each peripheral physiological response and experienced disgust was then performed by plotting each physiological variable against residuals from this general linear model with that variable removed.

\section{Results}

\section{Subjective ratings of disgust videos}

Ratings of core and BBV disgust videos confirmed that elicitors of core and BBV disgust induced equally potent subjective disgust (paired $t_{(11)}=0.01 ; p=0.99$ ). Mean (SD) disgust ratings of core and BBV disgust videos were 62.6 (14.2) and 55.3 (6.2), respectively. Core disgust videos also induced significantly greater concurrent feelings of nausea [paired $t_{(11)}=2.45 ; p=0.032$; mean (SD) nausea rating core and BBV videos were 56.6 (11.4) and 39.6 (6.0), respectively]; however, there was no increase in feelings of light-headedness/dizziness associated with BBV disgust videos $\left[t_{(11)}=0.67 ; p=0.52\right.$; mean (SD) faint rating core and BBV videos were 30.5 (5.4) and 32.7 (4.3), respectively].

As anticipated, high-intensity disgust videos induced significantly greater experienced disgust across modality (main effect of intensity; $\left.F_{(3,11)}=24.10 ; p<0.001\right)$. Importantly, there was no significant main effect of modality $\left(F_{(3,11)}=1.31 ; p=0.28\right)$ or interaction between modality and disgust intensity $\left(F_{(3,11)}=\right.$ $3.05 ; p=0.11)$, confirming that the core and BBV stimuli were of similar potencies.

\section{Peripheral physiology and experience of disgust}

Both cardiac and gastric responses correlated with the overall magnitude of experienced disgust (tachygastria: $F_{(1,4)}=38.8, p=$ 0.003; low-frequency cardiac power: $\left.F_{(1,4)}=22.5, p=0.009\right)$. Crucially, significant interactions between both gastric and cardiac responses and the form of experienced disgust were also observed (tachygastria: $F_{(1,4)}=33.6, p=0.004$; low-frequency cardiac power: $F_{(1,4)}=28.0, p=0.006$; high-frequency cardiac power: $\left.F_{(1,4)}=19.4, p=0.012\right)$. Visualization of the relationship between each peripheral physiological response and experienced disgust revealed a greater dependence of experienced core disgust on gastric (tachygastric) responses and experienced BBV disgust on parasympathetically mediated changes in the heart (see supplemental Fig. 1, available at www.jneurosci.org as supplemental material). Mean percentage power for core and BBV videos, respectively, were 28.2 (2.4) and 31.7 (3.3) for low-frequency cardiac power, 13.1 (2.6) and 14.5 (1.1) for high-frequency power, 35.3 (4.7) and 31.7 (3.2) for normogastria, and 9.6 (1.1) and 10.5 (1.7) for tachygastria.

\section{Neural correlations of experienced disgust}

Contrast of core versus BBV disgust videos (main effect of disgust type) revealed a differential pattern of insula activations to observation of elicitors of core and BBV disgust, with elicitors of core disgust activating a more ventral midanterior region and BBV disgust additionally activating primary sensory-motor cortices (Fig. 1 and supplemental Tables 1 and 2, available at www.jneurosci.org as supplemental material).

To determine regions sensitive to the intensity of experienced disgust, we next performed a parametric analysis of the fMRI data analogous to our physiological analyses. Greater subjectively experienced disgust, regardless of modality, was associated with activation of a matrix of previously reported disgust-related areas including posterior and anterior insula (Phillips et al., 1997), basal ganglia, thalamus, and bilateral somatosensory and somatomotor cortices (Calder et al., 2007) (Table 1, Fig. 2). Importantly, interactions between the magnitude of experienced disgust and disgust modality were also observed in the left and right insula, respectively, to the experience of BBV versus core disgust (Figs. 3, 4), suggesting that the experience of core or BBV disgust is associated with both a different pattern of peripheral physiological activity and central neural responses.

\section{Relationship between peripheral and central responses to experienced disgust}

We then addressed the question of how disgust-specific patterns of peripheral physiological responses relate to the neural responses underpinning the subjective experience of disgust. Analysis of the physiological data (described above) revealed that changes in gastric activity (and, to a lesser extent, sympathetically mediated changes in the heart) predicted the magnitude of experienced disgust regardless of disgust type. We therefore performed a conjunction analysis to identify brain regions whose activity correlated with both gastric physiological change and subjective experience of disgust. Strikingly, this revealed coactivated regions centered on the midanterior insula bilaterally, supporting our prediction that changes in peripheral physiology contribute to activation of central regions believed to encode a 


\section{A Core greater than BBV Disgust}
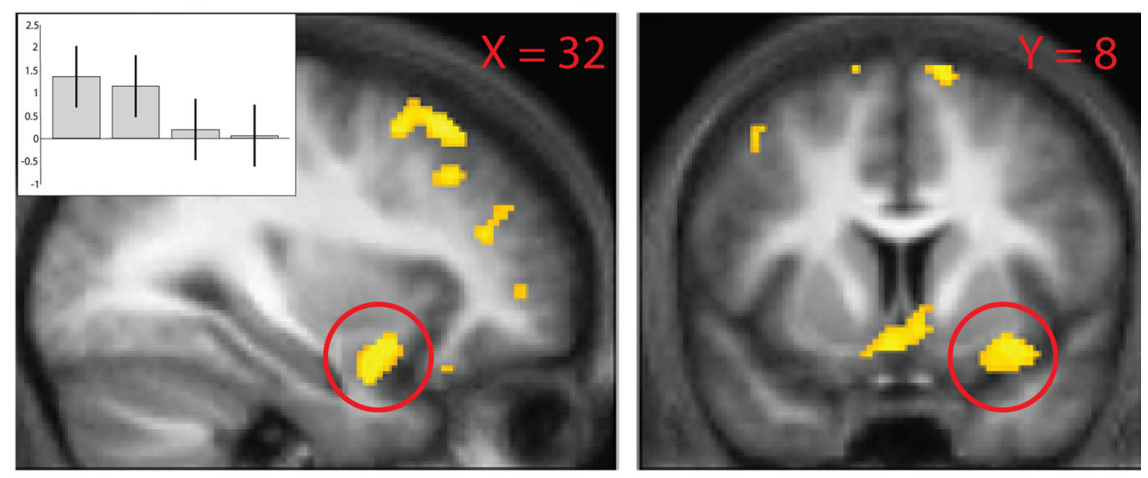

B BBV greater than Core disgust
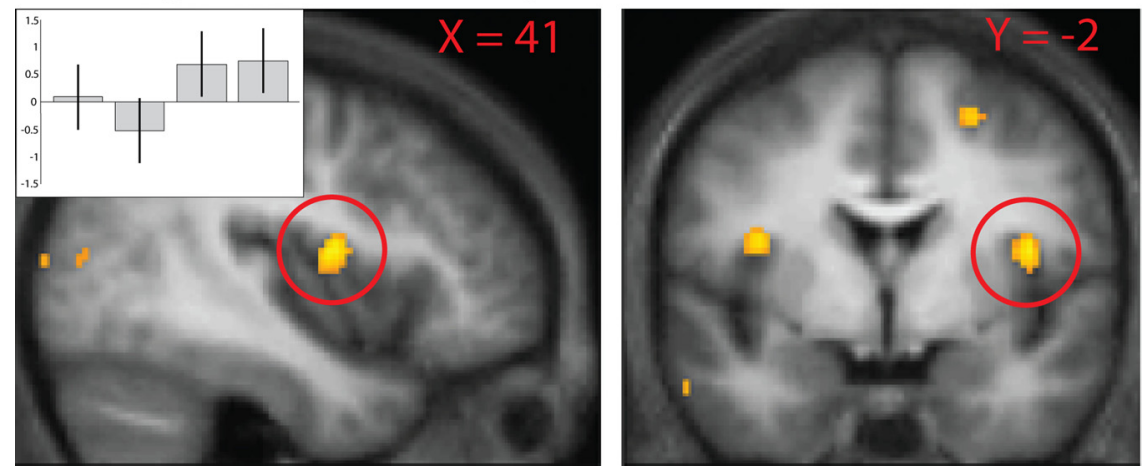

Figure 1. Insula activations to core and BBV disgust. $A$, Core greater than BBV disgust. $B, B B V$ greater than core disgust. Contrast estimates show activations in circled right ventral and dorsal insula, respectively, in order (left to right): high core, low core, high $\mathrm{BBV}$, and low BBV disgust. Activations are illustrated at $p<0.005$.

Table 1. Subjective experience of disgust ${ }^{a}$

\begin{tabular}{lrrrrrr}
\hline Side region (MNI) & \multicolumn{1}{c}{$y$} & \multicolumn{1}{c}{$z$} & Zscore & $k$ & Uncorrected $p$ \\
\hline L Anterior insula & $\mathbf{- 3 8}$ & $\mathbf{8}$ & $\mathbf{1 4}$ & $\mathbf{4 . 4 0}$ & $\mathbf{2 2 3}$ & $<\mathbf{0 . 0 0 1}$ \\
R Anterior insula & $\mathbf{3 4}$ & $\mathbf{8}$ & $\mathbf{2}$ & $\mathbf{4 . 2 9}$ & $\mathbf{1 8 7}$ & $<\mathbf{0 . 0 0 1}$ \\
L Anterior insula & $\mathbf{- 2 8}$ & $\mathbf{2 4}$ & $\mathbf{4}$ & $\mathbf{4 . 1 1}$ & $\mathbf{6 0}$ & $<\mathbf{0 . 0 0 1}$ \\
L Posterior insula & $\mathbf{- 3 0}$ & $\mathbf{- 1 6}$ & $\mathbf{1 6}$ & $\mathbf{3 . 4 3}$ & $\mathbf{7}$ & $<\mathbf{0 . 0 0 1}$ \\
R Primary motor cortex & 30 & -20 & 58 & 3.78 & 14 & $<0.001$ \\
L Primary motor cortex & -32 & -14 & 40 & 3.52 & 12 & $<0.001$ \\
L Postcentral gyrus & -42 & -18 & 44 & 3.40 & 16 & $<0.001$ \\
L Precuneus & -4 & -50 & 58 & 3.55 & 13 & $<0.001$ \\
L DLPFC & -38 & 42 & 32 & 3.53 & 14 & $<0.001$ \\
R DLPFC & 40 & 48 & 28 & 4.37 & 106 & $<0.001$ \\
R+L Dorsomedial & 0 & -2 & 4 & 3.53 & 22 & $<0.001$ \\
$\quad$ thalamus & & & & & & \\
L Putamen & -18 & 10 & -4 & 3.48 & 30 & $<0.001$ \\
\hline
\end{tabular}

${ }^{a}$ Brain regions correlating with the magnitude of experienced disgust across both modalities. Significant activations in the insula and corresponding cluster size are shown in bold. Extra-insula regions of $\geq 10$ contiguous voxels at an uncorrected $p<0.001$ are reported. L, Left; R, right; DLPFC, dorsolateral prefrontal cortex.

consciously accessible representation of disgust (Fig. 2, Table 2). Additional coactivated regions included the set of cortical and subcortical structures implicated in providing a central representation of physiological state including medial thalamus incorporating the mediodorsal nucleus and bilateral thalamic regions encompassing basal and posterior ventromedial nuclei, which project to dorsal mid/posterior insula (Craig, 2002). Interestingly, despite cardiac physiological change predicting the magnitude of experienced disgust, no brain region reflected changes in both cardiac physiology and experienced disgust across modalities.

Finally, we addressed the critical question of whether central representations of organ-specific physiological responses to the two disgust forms also underpinned differential emotional experience. Our physiological analysis had identified differential gastric and cardiac contributions to the experience of BBV versus core disgust (supplemental Fig. 1, available at www.jneurosci.org as supplemental material). Experience of BBV disgust was associated with a decrease in parasympathetic cardiac responses and, conversely, core disgust with an increase (i.e., a crossover interaction). We therefore looked for brain regions that showed both a crossover interaction with the form of experienced disgust and parasympathetically mediated influences on the heart. This conjunction analysis revealed common activations in only two discrete regions, the left anterior insula and right primary motor cortex (Fig. 3, Table 3).

Physiological analysis also showed a differential relationship between tachygastria and the form of disgust experienced (supplemental Fig. 1, available at www. jneurosci.org as supplemental material). In particular, tachygastria showed a stronger positive correlation with experience of core than BBV disgust (i.e., a noncrossover or simple interaction). To test for this simple interaction in tachygastric changes, we therefore identified brain regions coactivated by both the subjective experience of core disgust and tachygastric responses to core disgust elicitors. This conjunction analysis identified only three significantly activated regions, two within midanterior insula on the right and one in the cingulate cortex (Fig. 4, Table 4).

\section{Discussion}

Across these analyses, the magnitude of subjectively experienced disgust, regardless of disgust form, correlated with anterior insula activity. This finding is consistent with previous reports of anterior insula activity to experience of disgusting odors (Wicker et al., 2003) and observation of intense versus mildly disgusting facial expressions (Phillips et al., 1997) and suggests an important role for anterior insula in the experience of disgust regardless of form. Interestingly, data from lesion studies suggests that perception of disgust regardless of sensory modality may depend on the structural integrity of an alternate, more ventral insula region (Kipps et al., 2007), the anterior section of the anterior long insula gyrus. The importance of this ventral insula region to disgust perception is also supported by human depth electrode recordings showing a differential response of neurons in this region to implicit or explicit judgments of facial disgust expressions (Krolak-Salmon et al., 2003). Our own data showing ventral anterior insula activity to observation, but not subjective experience, of core versus BBV disgust extend these findings and suggest a specific role for ventral insula in the perception, but not subjective experience, of core-ingestive disgust.

Disgust rather than elicitors of other emotional states was chosen in this study for two reasons: (1) because disgust is often considered the most visceral of all basic emotions and (2) because the range of elicitors and corresponding subtleties in physiolog- 


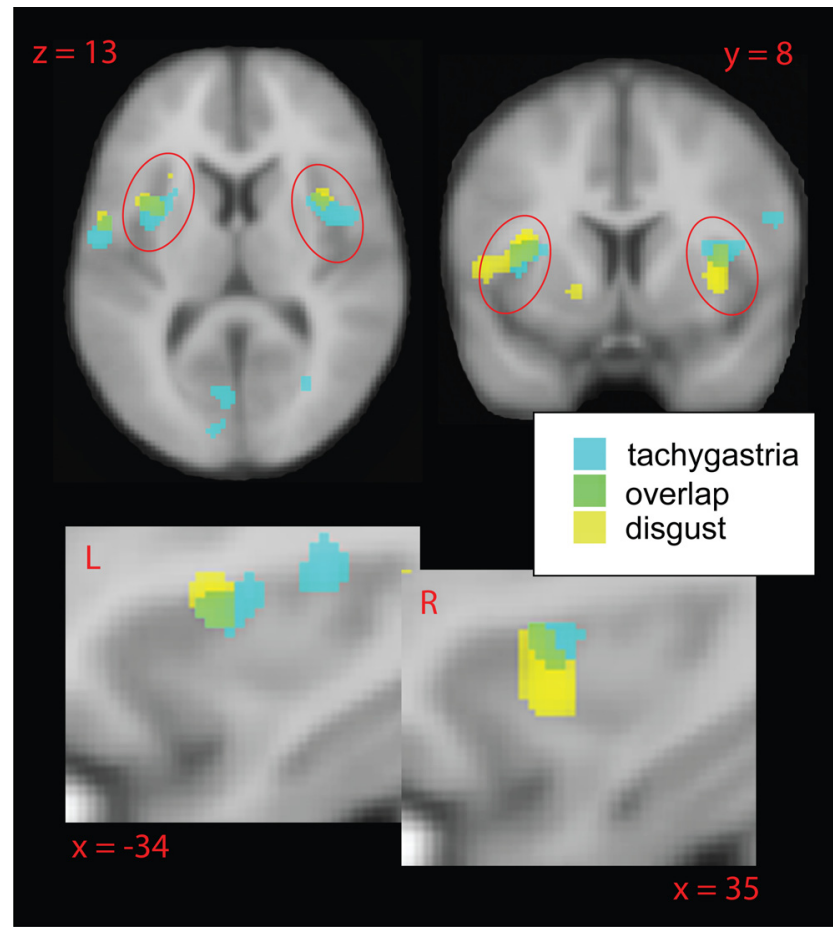

Figure 2. Conjunction of experienced disgust and tachygastria. Brain regions activated to experienced disgust (both core and BBV) are shown in yellow, and tachygastric response (both core and BBV) is shown in blue. (ommonly activated regions are illustrated in green. Activations are illustrated at $p<0.005$

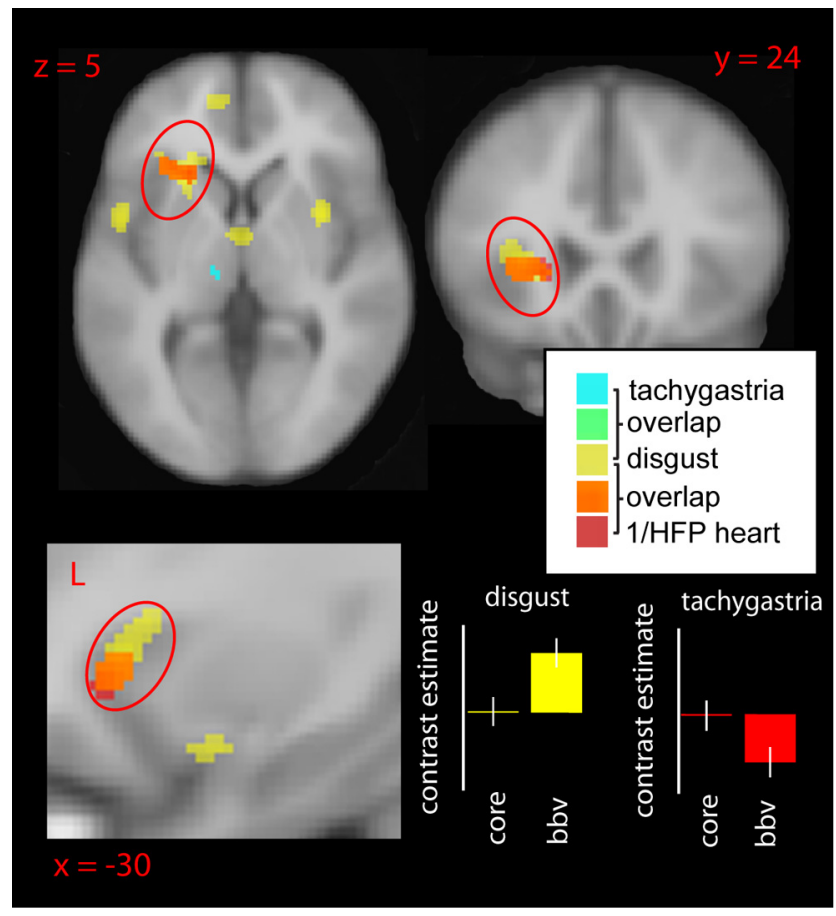

Figure 3. Conjunction of experienced BBV disgust and peripheral physiology. Shown are brain regions correlating with the magnitude of experienced BBV disgust (yellow) and 1/highfrequency (parasympathetic) cardiac power [1/HFP (high frequency power); red] and tachygastria (blue) with commonly activated regions to magnitude of BBV disgust (orange) and 1/HFP and tachygastria (green). Inset, Bar graphs show the contrast estimates ( $\pm 90 \%$ confidence interval) for the left dorsal anterior insula for (from left to right) experience of core disgust, experience of BBV disgust (both in yellow), and correlation with $1 / \mathrm{HFP}$ to core disgust and BBV disgust videos (both in red)

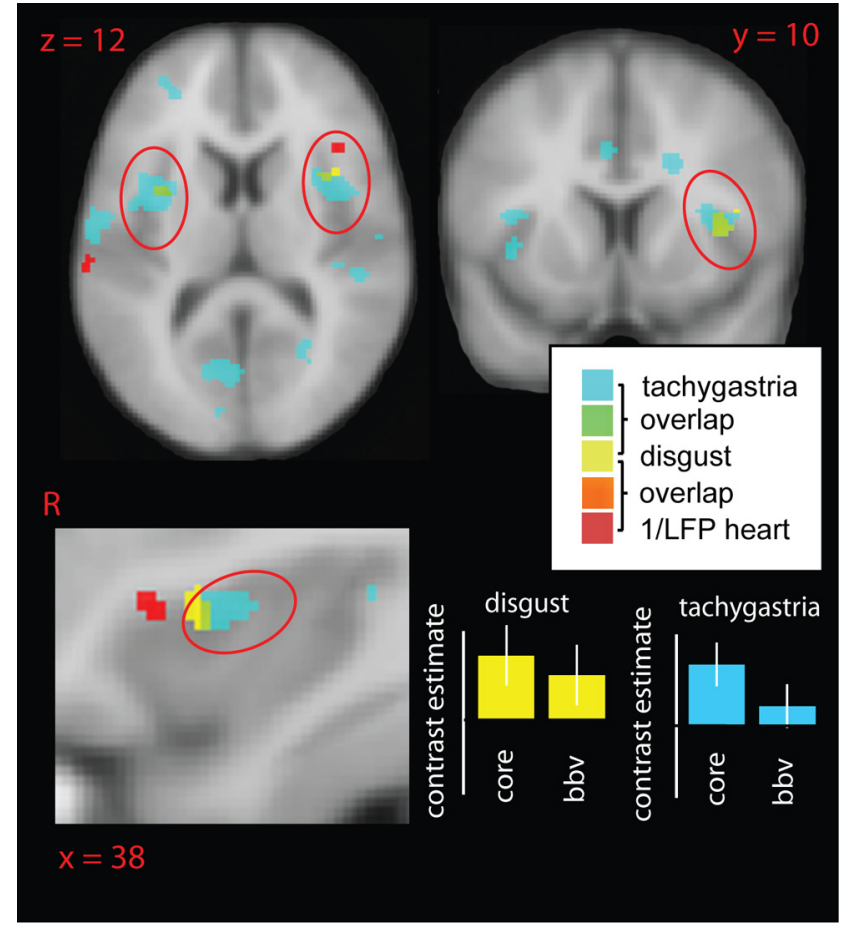

Figure 4. Conjunction of experienced core disgust and peripheral physiology. Shown are brain regions correlating with the magnitude of experienced core disgust (yellow) and 1/low frequency (sympathetic) cardiac power (1/LFP; red) and tachygastria (blue) with commonly activated regions to core disgust and 1/LFP (orange) and tachygastria (green). Inset, Bar graphs show the contrast estimates ( $\pm 90 \%$ confidence interval) for the right dorsal insula for (from left to right) experience of core disgust, experience of BBV disgust (both in yellow), and correlation with tachygastria to core disgust and BBV disgust videos (both in red).

ical responses to the experience of disgust are more carefully studied than for other basic emotions. For example, images of body waste products or ingestion of spoiled food are associated with distinct facial expressions (nose wrinkle, mouth gape, and tongue protrusion) and induction of "core disgust" (Rozin et al., 1994). In contrast, images of body mutilation are associated with upper lip retraction rather than nose wrinkle, gape, or tongue protrusion and induction of BBV disgust (Rozin et al., 1994).

Core-ingestive and BBV disgust also induce different patterns of neural activation. Although elicitors of both disgust forms activate anterior insula, BBV disgust has been additionally associated with superior parietal activation (Wright et al., 2004). Naturalistic exposure to these disgust elicitors is also associated with different experiential, behavioral, and physiological responses. BBV disgust elicitors (e.g., images of body mutilation, blood, or venesection) induce feelings of disgust accompanied by lightheadedness or dizziness and changes in cardiovascular responses, expressed in an extreme form in 3-4\% of the population as vasovagal syncope (Accurso et al., 2001). Subjects in the current study also rated greater light-headedness or dizziness to BBV videos, although this did not reach statistical significance, perhaps because of the fact that these videos were watched by subjects laying flat. Reports of retching or vomiting to these stimuli are rare. In contrast, core disgust elicitors (e.g., spoiled food or vomitus) induce feelings of disgust and nausea, retching, reduction in slow rhythmic gastric activity (bradygastria) and an increase in rapid dysregulated gastric responses known as tachygastria (Stern et al., 1989; Jokerst et al., 1997; Gianaros et al., 2001). This, together with an absence of reports of vaso-vagal syncope to core disgust 
Table 2. Conjunction of tachygastria and experienced disgust ${ }^{a}$

\begin{tabular}{|c|c|c|c|c|c|c|c|}
\hline Side region (MNI) & $x$ & $y$ & $z$ & Zscore & $k$ & Uncorrected $p$ & $\mathrm{FDR} p$ \\
\hline L Posterior insula & -30 & -16 & 20 & 4.67 & 45 & $<0.001$ & $<0.003$ \\
\hline$L+R$ aMCC/pMCC & 0 & 0 & 36 & 4.41 & 106 & $<0.001$ & $<0.003$ \\
\hline L Precentral gyrus & -38 & -10 & 44 & 4.20 & 217 & $<0.001$ & $<0.003$ \\
\hline L Anterior insula & -36 & 2 & 10 & 4.01 & 204 & $<0.001$ & $<0.003$ \\
\hline R Anterior insula & 30 & 10 & 14 & 4.00 & 221 & $<0.001$ & $<0.003$ \\
\hline L Insula/SII & -50 & -6 & 4 & 3.92 & 220 & $<0.001$ & $<0.003$ \\
\hline L Thalamus & -12 & -18 & 0 & 3.90 & 14 & $<0.001$ & $<0.003$ \\
\hline L Frontal lobe & -14 & -6 & 60 & 3.67 & 17 & $<0.001$ & $<0.003$ \\
\hline L Occipital lobe & -16 & -80 & 28 & 3.45 & 16 & $<0.001$ & $<0.004$ \\
\hline L Frontal lobe & -16 & -10 & 70 & 3.34 & 10 & $<0.001$ & $<0.005$ \\
\hline R Fusiform gyrus & 38 & -66 & -18 & 3.04 & 20 & $<0.001$ & $<0.008$ \\
\hline RDM thalamus & 6 & -22 & 16 & 2.97 & 14 & $<0.001$ & $<0.009$ \\
\hline L Parietal lobe & -36 & -48 & 36 & 2.96 & 37 & $<0.002$ & $<0.009$ \\
\hline RThalamus & 16 & -12 & 8 & 2.86 & 10 & $<0.002$ & $<0.011$ \\
\hline
\end{tabular}

${ }^{a}$ Conjunction performed by masking activations to tachygastria (threshold, $p<0.005$ ) with activations to experienced disgust (threshold, $p<0.005$ ). Regions of $\geq 10$ contiguous voxels at FDR $<0.05$ reported. aMCC, Anterior medial cingulate cortex; PMCC, posterior cingulate cortex; DM, dorsomedial.

Table 3. Conjunction of experienced core disgust and tachygastria ${ }^{a}$

\begin{tabular}{lllllll}
\hline Side region (MNI) & $x$ & $y$ & $z$ & Zscore & $k$ & Uncorrected $p$ \\
\hline L Dorsal midinsula & -36 & 4 & 12 & 4.56 & 22 & $<0.001$ \\
R Dorsal anterior insula & 34 & 10 & 10 & 3.70 & 35 & $<0.001$ \\
LaMCC & -12 & 24 & 30 & 2.96 & 10 & $<0.002$ \\
\hline
\end{tabular}

${ }^{a}$ Conjunction performed by masking activations to tachygastria (threshold, $p<0.001$ ) with activations to intensity of experienced core disgust (threshold, $p<0.005$ ). No region significantly correlated with core disgust and normogastria/ cardiac high frequency power/cardiac low frequency power or inversely with tachygastria. aMCC, Anterior medial cingulate cortex.

Table 4. Conjunction of experienced boundary-violation disgust and inverse HFP ${ }^{a}$

\begin{tabular}{lrrrrrr}
\hline Side region (MNI) & $x$ & $y$ & $z$ & Zscore & $k$ & Uncorrected $p$ \\
\hline R Primary motor cortex & 32 & -20 & 60 & 3.79 & 15 \\
L Anterior insula & -30 & 26 & 2 & 3.19 & $<0.001$ & 23 \\
\hline
\end{tabular}

${ }^{a}$ Conjunction performed by masking activations to inverse HFP (threshold, $p<0.001$ ) with activations to intensity of experienced BBV disgust (threshold, $p<0.005$ ). No regions correlated with BBV disgust and normogastria, cardiac low frequency power, positively with cardiac high frequency power (HFP), or inversely with tachygastria.

elicitors, represents a dissociation of experiential and physiological responses to these two distinct disgust forms.

One interpretive consideration with conjunction analyses is whether brain regions showing common activations to both physiological responses and the experience of disgust truly represent a common neural substrate and interface between embodied and experiential processes or, alternately, whether the activations reflect the neural representation of one process (e.g., declarative experience) that indirectly correlates with the second process (physiological state). Were the latter interpretation the case, we would anticipate that the majority of activations to either physiological state or experiential state would colocalize. In fact, in our data, coactivations [which were false discovery rate (FDR) whole-brain corrected] represented only $1.2-6.7 \%$ of all activations to physiological or experiential state alone, strongly supporting our claim that anterior insula regions represent a common neural substrate for embodied and experiential processes.

An important consideration in the interpretation of all studies relating peripheral physiological and brain imaging data is whether observed neural responses relate to afferent or efferent representations of physiological activity (Critchley et al., 2000). As here, technical constraints on the ability to directly measure afferent autonomic activity necessitate the use of indirect measures indexed by efferent physiological activity. Our interpretation of insula activations as afferent representations of disgust-related physiological change are consistent with human stimulation (Penfield and Faulk, 1955) and functional imaging studies (Craig, 2002) demonstrating somatotopically organized representations of bodily physiological state within insula.

However, it is also conceivable that our observed insula activations may result not from direct representations of peripheral physiological activity but from centrally generated efference copies of autonomic motor discharge. Efference copy or corollary discharge of motor signals are predicted to be widely used within the somatic motor system (Sommer and Wurtz, 2008), serving, among other roles, to predict the sensory consequences of internally generated motor actions. Similar computational challenges (e.g., a need to differentiate central from peripherally initiated changes in body physiological state) suggest a similar neural architecture may exist within the autonomic nervous system (Damasio, 1994). Presence of efference copies of centrally generated changes to body physiology are also likely to be rapidly generated, potentially addressing another of Cannon's criticisms of somatic theories of emotion: that peripheral physiological changes are too slow to account for associated changes in subjective feelings (Cannon, 1931).

Regardless of the proximate cause of insula activations, our findings suggest a dissociation in both peripheral physiological and central neural responses to two discrete forms of experienced disgust. Experience of core disgust was associated with an increase in tachygastric responses in the stomach and right anterior insula activity, whereas BBV disgust was associated with a reduction in parasympathetically mediated influences on the heart and activity in the left anterior insula. Strikingly, peripheral physiological changes predicted these central neural patterns, suggest- 
ing that differential peripheral physiological responses directly contribute to subjective emotional experience and provide valuable support for somatic theories of emotion.

\section{References}

Accurso V, Winnicki M, Shamsuzzaman ASM, Wenzel A, Johnson AK, Somers VK (2001) Predisposition to vasovagal syncope in subjects with blood/injury phobia. Circulation 104:903-907.

Andrew D, Craig AD (2001a) Spinothalamic lamina I neurones selectively responsive to cutaneous warming in cats. J Physiol 537:489-495.

Andrew D, Craig AD (2001b) Spinothalamic lamina I neurons selectively sensitive to histamine: a central neural pathway for itch. Nat Neurosci 4:72-77.

Berntson GG, Bigger JT, Eckberg DL, Grossman P, Kaufmann PG, Malik M, Nagaraja HN, Porges SW, Saul JP, Stone PH, VanderMolen MW (1997) Heart rate variability: origins, methods, and interpretive caveats. Psychophysiology 34:623-648.

Cacioppo JT, Berntson GG, Larsen JT (2000) The psychophysiology of emotion. In: The handbook of emotion (Lewis M, Haviland-Jones JM, eds), pp 173-191. New York: Guilford.

Calder AJ, Beaver JD, Davis MH, van DJ, Keane J, Lawrence AD (2007) Disgust sensitivity predicts the insula and pallidal response to pictures of disgusting foods. Eur J Neurosci 25:3422-3428.

Cannon WB (1931) Again the James-Lange and the thalamic theories of emotion. Psychol Rev 38:281-295.

Christie IC, Friedman BH (2004) Autonomic specificity of discrete emotion and dimensions of affective space: a multivariate approach. Int J Psychophysiol 51:143-153.

Collet C, VernetMaury E, Delhomme G, Dittmar A (1997) Autonomic nervous system response patterns specificity to basic emotions. J Autonom Nervous Syst 62:45-57.

Craig AD (2002) How do you feel? Interoception: the sense of the physiological condition of the body. Nat Rev Neurosci 3:655-667.

Critchley HD, Elliott R, Mathias CJ, Dolan RJ (2000) Neural activity relating to generation and representation of galvanic skin conductance responses: a functional magnetic resonance imaging study. J Neurosci 20:3033-3040.

Critchley HD, Wiens S, Rotshtein P, Ohman A, Dolan RJ (2004) Neural systems supporting interoceptive awareness. Nat Neurosci 7:189-195.

Damasio AR (1994) Descartes' error: emotion, reason and the human brain. New York: Grosset/Putnam.

Damasio AR, Grabowski TJ, Bechara A, Damasio H, Ponto LLB, Parvizi J, Hichwa RD (2000) Subcortical and cortical brain activity during the feeling of self-generated emotions. Nat Neurosci 3:1049-1056.

Deichmann R, Gottfried JA, Hutton C, Turner R (2003) Optimized EPI for fMRI studies of the orbitofrontal cortex. Neuroimage 19:430-441.

Ekman P, Levenson RW, Friesen WV (1983) Autonomic nervous-system activity distinguishes among emotions. Science 221:1208-1210.

Gianaros PJ, Quigley KS, Mordkoff JT, Stern RM (2001) Gastric myoelectrical and autonomic cardiac reactivity to laboratory stressors. Psychophysiology 38:642-652.

James W (1894) Physical basis of emotion. Psychol Rev 1:516-529.

Janig W (2008) Integrative action of the autonomic nervous system: neurobiology of homeostasis. Cambridge, UK: Cambridge UP.

Jokerst MD, Levine M, Stern RM, Koch KL (1997) Modified sham feeding with pleasant and disgusting foods: cephalic-vagal influences on gastric myoelectric activity. Gastroenterology 112:A755.

Jones CL, Ward J, Critchley HD (2010) The neuropsychological impact of insular cortex lesions. J Neurol Neurosurg Psychiatry 81:611-618.

Kikyo H, Ohki K, Miyashita Y (2002) Neural correlates for feeling-of-knowing: an fMRI parametric analysis. Neuron 36:177-186.

Kipps CM, Duggins AJ, McCusker EA, Calder AJ (2007) Disgust and happiness recognition correlate with anteroventral insula and amygdala volume respectively in preclinical Huntington's disease. J Cogn Neurosci 19:1206-1217.

Krolak-Salmon P, Henaff MA, Isnard J, Tallon-Baudry C, Guenot M, Vighetto A, Bertrand O, Mauguiere F (2003) An attention modulated response to disgust in human ventral anterior insula. Ann Neurol 53:446-453.

Levenson RW, Ekman P, Friesen WV (1990) Voluntary facial action generates emotion-specific autonomic nervous-system activity. Psychophysiology 27:363-384.

Morrison SF (2001) Differential control of sympathetic outflow. Am J Physiol 281:R683-R698.

Mufson EJ, Mesulam MM (1982) Insula of the old-world monkey. 2. Afferent cortical input and comments on the claustrum. J Comp Neurol 212:23-37.

Naqvi NH, Rudrauf D, Damasio H, Bechara A (2007) Damage to the insula disrupts addiction to cigarette smoking. Science 315:531-534.

Penfield W, Faulk ME (1955) The insula: further observations on its function. Brain 78:445-470.

Phillips ML, Young AW, Senior C, Brammer M, Andrew C, Calder AJ, Bullmore ET, Perrett DI, Rowland D, Williams SCR, Gray JA, David AS (1997) A specific neural substrate for perceiving facial expressions of disgust. Nature 389:495-498.

Porges SW (2007) The polyvagal perspective. Biol Psychol 74:116-143.

Rainville P, Bechara A, Naqvi N, Damasio AR (2006) Basic emotions are associated with distinct patterns of cardiorespiratory activity. Int J Psychophysiol 61:5-18.

Rozin P, Lowery L, Ebert R (1994) Varieties of disgust faces and the structure of disgust. J Pers Soc Psychol 66:870-881.

Sommer MA, Wurtz RH (2008) Brain circuits for the internal monitoring of movements. Annu Rev Neurosci 31:317-338.

Stern RM (2002) The psychophysiology of nausea. Acta Biol Hungarica 53:589-599.

Stern RM, Crawford HE, Stewart WR, Vasey MW, Koch KL (1989) Sham feeding - cephalic-vagal influences on gastric myoelectric activity. Digest Dis Sci 34:521-527.

Vernet-Maury E, Alaoui-Ismaili O, Dittmar A, Delhomme G, Chanel J (1999) Basic emotions induced by odorants: a new approach based on autonomic pattern results. J Autonom Nervous Syst 75:176-183.

Wicker B, Keysers C, Plailly J, Royet JP, Gallese V, Rizzolatti G (2003) Both of us disgusted in my insula: the common neural basis of seeing and feeling disgust. Neuron 40:655-664.

Winston JS, Strange BA, O'Doherty J, Dolan RJ (2002) Automatic and intentional brain responses during evaluation of trustworthiness of faces. Nat Neurosci 5:277-283.

Wright P, He G, Shapira NA, Goodman WK, Liu Y (2004) Disgust and the insula: fMRI responses to pictures of mutilation and contamination. Neuroreport 15:2347-2351. 